\title{
Direct-acting antivirals-based therapy decreases hepatic fibrosis serum biomarker microfibrillar- associated protein 4 in hepatitis C patients
}

\author{
Christian Mölleken', Maike Ahrens ${ }^{2,3}$, Anders Schlosser $^{4}$, Julia Dietz $^{5}$, Martin Eisenacher ${ }^{2}$, Helmut E. Meyer ${ }^{2,6}$, \\ Wolff Schmiegel', Uffe Holmskov ${ }^{4}$, Christoph Sarrazin ${ }^{5,7}$, Grith Lykke Sorensen ${ }^{4}$, Barbara Sitek $^{2}$, and \\ Thilo Bracht ${ }^{2}$ \\ 'Department of Gastroenterology and Hepatology, University Hospital Bergmannsheil, Bochum; ${ }^{2}$ Medizinisches Proteom-Center, Ruhr \\ University Bochum, Bochum; ${ }^{3}$ Chrestos Concept GmbH \& Co. KG, Essen, Germany; ${ }^{4}$ Institute for Molecular Medicine, University of \\ Southern Denmark, Odense, Denmark; ${ }^{5}$ Medical Clinic 1, J.W. Goethe University Hospital, Frankfurt; ${ }^{6}$ Leibniz-Institut für Analytische \\ Wissenschaften - ISAS - e.V., Dortmund; ${ }^{7}$ Medical Clinic 2, St. Josefs-Hospital, Wiesbaden, Germany
}

Background/Aims: An estimated 80 million people worldwide are infected with viremic hepatitis $C$ virus (HCV). Even after eradication of HCV with direct acting antivirals (DAAs), hepatic fibrosis remains a risk factor for hepatocarcinogenesis. Recently, we confirmed the applicability of microfibrillar-associated protein 4 (MFAP4) as a serum biomarker for the assessment of hepatic fibrosis. The aim of the present study was to assess the usefulness of MFAP4 as a biomarker of liver fibrosis after HCV eliminating therapy with DAAs.

Methods: MFAP4 was measured using an immunoassay in 50 hepatitis $C$ patients at baseline (BL), the end-of-therapy (EoT), and the 12-week follow-up (FU) visit. Changes in MFAP4 from BL to FU and their association with laboratory parameters including alanine aminotransferase (ALT), aspartate aminotransferase (AST), platelets, the AST to platelet ratio index (APRI), fibrosis-4 score (FIB-4), and albumin were analyzed.

Results: MFAP4 serum levels were representative of the severity of hepatic fibrosis at BL and correlated well with laboratory parameters, especially APRI (Spearman correlation, $\mathrm{R}^{2}=0.80$ ). Laboratory parameters decreased significantly from BL to EoT. MFAP4 serum levels were found to decrease from BL and EoT to FU with high statistical significance (Wilcoxon $P<0.001$ for both).

Conclusions: Our findings indicate that viral eradication resulted in reduced MFAP4 serum levels, presumably representing a decrease in hepatic fibrogenesis or fibrosis. Hence, MFAP4 may be a useful tool for risk assessment in hepatitis C patients with advanced fibrosis after eradication of the virus. (Clin Mol Hepatol 2019;25:42-51)

Keywords: Hepatitis C, Chronic; Biomarkers; Liver cirrhosis; Antiviral agents; Extracellular matrix proteins

\footnotetext{
Abbreviations:

ALT, alanine aminotransferase; APRI, aspartate aminotransferase to platelet ratio index; AST, aspartate aminotransferase; DAA, direct acting antiviral; ECM, extracellular matrix; EoT, end-of-therapy; FIB-4, fibrosis-4 score; FU, followup; F0-F4, hepatic fibrosis stages 0-4 (METAVIR); HCV, hepatitis C virus; IFN-a, interferon-a; METAVIR, scoring system for the assessment of hepatic fibrosis; MFAP4, microfibrillar-associated protein 4; SVR, sustained virologic response; TE, transient elastography
}

Corresponding author : Thilo Bracht

Medizinisches Proteom-Center, Ruhr University Bochum, D-44802

Bochum, Germany

Tel: +49-234-32-29985

E-mail: thilo.bracht@rub.de

https://orcid.org/0000-0002-1194-6614 


\section{Study Highlights}

- We assessed the performance of microfibrillar-associated protein 4 (MFAP4) as a serum biomarker for hepatic fibrosis in 50 hepatitis C virus patients undergoing direct-acting antivirals therapy at baseline, the end-of-therapy, and the 12-week follow-up visit.

- MFAP4 serum levels were representative of the severity of hepatic fibrosis at baseline and correlated well with laboratory parameters, especially the aspartate aminotransferase to platelet ratio index (APRI).

- MFAP4 serum levels decreased from baseline and from the end-of-therapy to the 12-week follow-up visit with high statistical significance, indicating a decrease in hepatic fibrogenesis or fibrosis.

\section{INTRODUCTION}

There are estimated 80 million people with viremic hepatitis C virus (HCV) infection (corresponding to about $1 \%$ of the population) worldwide.' The acute HCV infection is typically asymptomatic and, in people who progress to chronic HCV infection, often unapparent until they develop significant liver damage years later. Besides an inflammatory response in some patients the HCV impairs the metabolism of infected hepatocytes. Moreover, viral proteins, e.g. core, nonstructural protein 3 (NS3), and nonstructural protein 5A (NS5A) can induce a disturbance of lipid metabolism and modulate hepatocyte apoptosis or alter signal transduction. ${ }^{2-5}$ The predominant profibrogenic cytokine is the transforming growth factor beta (TGFß-1), which can be released from almost any cell type during inflammation, tissue regeneration, and fibrogenesis. ${ }^{6-8}$ It strongly up-regulates the production and deposition of extracellular matrix (ECM) proteins like collagens, matrix metalloproteinases, and tissue inhibitors of metalloproteinases by myofibroblastic cells. These either derive from activated hepatic stellate cells or activated portal or perivascular fibroblasts. ${ }^{9,10}$ Therapy with interferon (IFN)-a has been reported to have antifibrotic effects by reducing the serum levels of fibrotic markers, such as the $\mathrm{N}$-terminal peptide of procollagen type III. ${ }^{11,12}$ Fortunately, the treatment of HCV infection was revolutionized with the development of direct-acting antiviral agents (DAAs). ${ }^{13-16}$ Within the current decade highly tolerable single daily dosing oral regimens, lasting 8-12 weeks and yielding cure rates of $>90 \%$ should become standard - renouncing on IFN containing regimens with several side effects. ${ }^{14}$

Increasing evidence suggests that hepatic fibrosis is reversible and can be reduced in patients with HCV-associated cirrhosis and sustained virologic response (SVR) after IFN-free antiviral therapy. This hypothesis is confirmed by the fact that liver stiffness in transient elastography (TE) measurements is diminished after DAA therapy, probably due to reduced necroinflammation after viral eradication and in consequence a regression of hepatic fibrosis. ${ }^{17}$ In order to detect new biomarkers for hepatic fibrosis, we previ- ously performed a proteomics analysis of microdissected cirrhotic septae and liver parenchyma cells. ${ }^{18}$ An elevated abundance of the microfibrillar-associated protein 4 (MFAP4) in the cirrhotic septae was detected and the measurement of MFAP4 serum levels in HCV patients by enzyme-linked immunosorbent assay (ELISA) demonstrated its applicability as a blood-based biomarker for non-invasive assessment of liver fibrosis. Recently, we confirmed the elevated abundance of MFAP4 in hepatic fibrosis in tissue samples from a large patient cohort on transcript and protein level..$^{19}$ MFAP4 is an oligomeric ECM protein that is expressed in association with elastic fibers in the entire body, especially in elastin rich tissues like the heart or the lung. ${ }^{20}$ It has been demonstrated to interact with Tropoelastin and Fibrillin during formation of elastic fibers ${ }^{21}$ and was found to be involved in smooth muscle cell activation in vascular and pulmonary remodeling. ${ }^{22,23}$ MFAP4 serum levels have been shown to correlate with pulmonary and cardiovascular diseases. ${ }^{20,24-26}$ but the most comprehensive evidence for an applicability of MFAP as biomarker has been presented for hepatic fibrosis. MFAP4 plasma levels were shown to vary in parallel with TE measurements and are significantly increased in patients with chronic HCV infection in support of previous observations. ${ }^{27}$ In a large retrospective study we demonstrated MFAP4 serum levels to be highly significantly increased from no to moderate fibrosis stages $\mathrm{F} 0-\mathrm{F} 2$ to severe stages ( $\mathrm{F} 3$ and $\mathrm{F} 4$ ) as determined by liver biopsy. We derived a multivariate model considering also age and gender which yielded $81.3 \%$ sensitivity and $61.5 \%$ specificity for the correct identification of $\mathrm{F} 3$ and F4. For the correct prediction of no to moderate fibrosis and severe fibrosis and cirrhosis the model performed with an area under the curve (AUC) of $0.831 .^{28}$

To complement studies using parameters like TE and hepatic venous wedge pressure (HVPG) the aim of the present study was to assess an potential antifibrotic effect of DAAs by analyzing MFAP4 serum levels at baseline (BL), end-of-therapy (EoT) and follow-up (FU) in $50 \mathrm{HCV}$-patients undergoing DAA therapy. 


\section{PATIENTS AND METHODS}

\section{Clinical cohort}

Serum samples from $n=50$ patients suffering from $\mathrm{HCV}$ and undergoing therapy with DAAs (collected by the Department of Gastroenterology and Hepatology, University Clinic of Frankfurt, Germany) were used for the analysis of MFAP4 serum levels. All investigations were performed according to the Declaration of Helsinki and the usage of patient blood samples and retrospective collection of data was approved by the local ethics committee (Ethics Committee of the J.W. Goethe University Frankfurt, reference number 16/15). Fifteen patients were known to be treatment-naive while 29 received treatment prior to the DAA therapy. MFAP4 levels were determined at baseline (BL, 0 weeks), EOT (12, 24 or 48 weeks) and FU time points (12 weeks after EoT). The corresponding histological staging by liver biopsy before therapy was available for $n=21$ patients. Laboratory parameters aspartate aminotransferase (AST), alanine aminotransferase (ALT), platelet count and albumin were available for BL and EoT time points. All available patients' data as well as laboratory data are summarized in Supplementary Table 1.

\section{Histologic staging}

Biopsies were staged blindly according to the scoring system for the assessment of hepatic fibrosis (METAVIR) by one pathologist with a specialization in liver pathology. Every biopsy specimen was staged on a scale of Fibrosis F0 to F4: F0 - no fibrosis; F1 enlarged, fibrotic portal tracts; F2 - periportal fibrosis or portalportal septa, but intact architecture; F3 - fibrosis with architectural distortion, but no obvious cirrhosis; and F4 - probable or definite cirrhosis.

\section{Measurement of MFAP4 serum concentrations}

The AlphaLISA technique (PerkinElmer) was used to measure MFAP4 serum levels as described before. ${ }^{20}$ Briefly, two monoclonal anti-MFAP4 antibodies (HG-HYB 7-14 and HG-HYB 7-18) that were generated using MFAP4-knock out mice were used as donor bead-coupled antibodies and acceptor bead coupled-antibodies, respectively. ${ }^{29}$ The measurements were performed in 384 well format and all sera were tested in duplicates diluted 1:100. When being measured in serum samples $1 \mathrm{U} / \mathrm{mL}$ MFAP4 corresponds to $38 \mathrm{ng} / \mathrm{mL}$ of MFAP4.

\section{AST to platelet ratio index (APRI) and fibrosis-4 score (FIB-4)}

The APRI was calculated as follows:

$$
A P R I=\left|\frac{A S T\left[\frac{U}{L}\right]}{\frac{\text { upper limit of normal AST }\left[\frac{\mathrm{U}}{\mathrm{L}}\right]}{\text { platelet count }\left[\frac{\times 1,000}{\mu \mathrm{L}}\right]}}\right| \times 100
$$

The considered upper limit of normal AST was 50 (U/L) for men and 35 (U/L) for women, respectively. The FIB- $4^{30}$ was calculated as follows:

$$
\text { FIB- } 4=\frac{\text { age }(\text { years }) \times \operatorname{AST}\left[\frac{\mathrm{U}}{\mathrm{L}}\right]}{\text { platelets }\left[\frac{\times 1,000}{\mu \mathrm{L}}\right] \times \operatorname{ALT}\left[\frac{\mathrm{U}}{\mathrm{L}}\right]}
$$

\section{Statistical analysis}

For the laboratory parameters and consequently also APRI and FIB-4 only data for BL and EoT time points were available. These were tested for a statistically significant difference using the Wilcoxon test. Correlation analysis of BL values for laboratory parameters, APRI and MFAP4 serum levels was performed using the Spearman's correlation coefficient. MFAP4 levels for BL, EOT and FU time points were analyzed using the Friedman test followed by a paired Wilcoxon test in case of a significant result of the Friedman test (post-hoc test). The resulting $P$-values were corrected for multiple testing using the Bonferroni approach. A significance level of $5 \%$ was chosen throughout the study.

\section{RESULTS}

\section{Baseline levels of MFAP4 and TE measurements reflect the severity of fibrosis}

For 21 patients histological staging according to the METAVIR classification was available. For 20 of those patients also data from TE measurements (fibroscan) were available. As a proof of concept, we divided the patients into two groups of no to moderate fibrosis stages F0-F2 $(n=9)$ and severe fibrosis and cirrhosis stages $F 3$ and F4 $(n=11)$. For both, MFAP4 and fibroscan a clear trend towards higher values in stages $\mathrm{F} 3$ and $\mathrm{F} 4$ was evident (Fig. 1). For fibroscan this was statistically significant (Wilcoxon $P$-value 0.04). For MFAP4 the difference was not significant due to an ap- 
parent outlier in F3, F4 (Wilcoxon $P$-value $0.08, P$-value after outlier exclusion 0.03). MFAP4 and fibroscan correlated significantly with $\mathrm{R}^{2}=0.66$ ( $P$-value $=0.002$, Supplementary Table 2).

\section{Correlation analysis of baseline levels of MFAP4 and laboratory parameters}

For all 50 patients the baseline levels of MFAP4 and the available laboratory parameters as well as APRI and FIB-4 were analyzed using the Spearman correlation method (Fig. 2, Supplementary Table 2). A high correlation of MFAP4 was found for AST $\left(\mathrm{R}^{2}=0.72, P\right.$-value $<1.13 \cdot 10^{-7}$ ) whereas the correlation to ALT was rather weak $\left(R^{2}=0.33\right.$, not significant [n.s.]). A negative correlation was observed for platelets and albumin (both $R^{2}=-0.67, P$-values $2.53 \cdot 10^{-6}$ and $1.69 \cdot 10^{-5}$, respectively). The highest correlation for MFAP4 was found with APRI $\left(R^{2}=0.80, P\right.$-value $\left.6.24 \cdot 10^{-11}\right)$. Amongst the other parameters the positive correlation of APRI and AST was found to be higher $\left(R^{2}=0.88, P\right.$-value $\left.1.29 \cdot 10^{-15}\right)$ which was obvious because APRI is a function of AST and platelet count. The same was true for FIB-4 which showed a high negative correlation with the platelet count $\left(R^{2}=-0.88, P\right.$-value $\left.7.75 \cdot 10^{-16}\right)$ but however was not significantly correlated with AST or ALT. FIB-4 and MFAP4 showed a weak correlation which was significant though $\left(R^{2}=0.64, P\right.$-value $\left.1.70 \cdot 10^{-5}\right)$.

\section{Laboratory parameters and the APRI decrease from baseline to the EoT}

The changes in AST and ALT levels between BL and EoT showed a significant decrease in all patients, both for the patient group with no to moderate (F0-F2) and severe fibrosis and cirrhosis (F3, F4), respectively (Fig. 3, Table 1). Median values dropped from 66 (U/L) and $67 \mathrm{U} / \mathrm{L}$ at BL to $25 \mathrm{U} / \mathrm{L}$ and $21.5 \mathrm{U} / \mathrm{L}$ at EoT for AST and ALT, respectively (Table 2). The platelet count increased in all patients with high statistical significance $\left(P\right.$-value $\left.9.65 \cdot 10^{-6}\right)$. The median platelet count increased from $151 \times 1,000 / \mu \mathrm{L}$ at $\mathrm{BL}$ to $170 \times 1,000 / \mu \mathrm{L}$ at EoT. When analyzing the subsets of patients with known fibrosis stages separately the increase of platelet counts was seen by trend but was not statistically significant. A statistically significant decrease of the APRI was observed in all patients, also when patients with $\mathrm{F} 0-\mathrm{F} 2$ and $\mathrm{F} 3$, F4 were analyzed separately. For F0-F2 the effect was more pronounced $(P$-value 0.004 ) than for F3 and F4 ( $P$-value 0.01$)$ and patients with severe fibrosis remained with a higher APRI at EoT. The median APRI in all patients decreased from 0.92 at BL to 0.32 at EoT. Albumin values increased from BL to EoT which was statistically significant when analyzing all patients. The median albumin proportion increased from 4.3 at BL to 4.45 at EoT. FIB-4 showed a moderate increase from 0.3 at BL to 3.4 at EoT ( $P$-value 0.03 ) when examining all patients. For patients with low fibrosis stages the increase was more evident whereas for high fibrosis stages even a decrease was evident. However, the median values were quite dis-

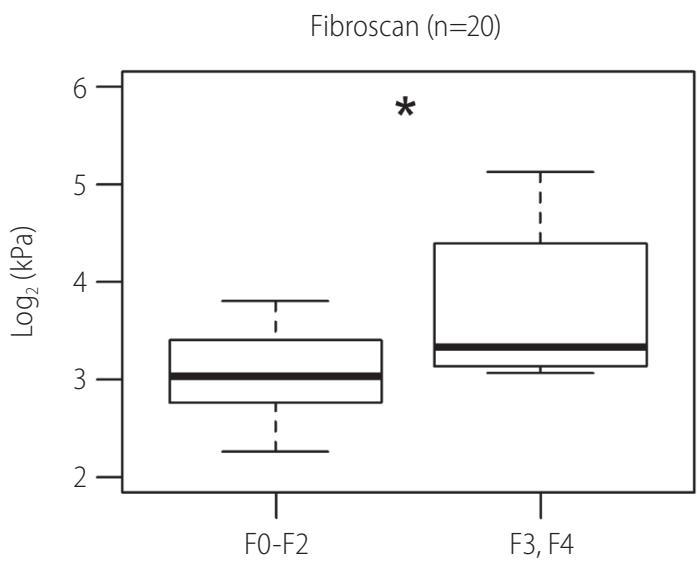

Figure 1. Baseline values of MFAP4 (A) and fibroscan (B) for no-to-moderate (F0-F2) and severe (F3, F4) fibrosis stages. The upper and lower bounds of boxes represent the first and third quartiles per group, whiskers extend to the most extreme data point, which is no more than 1.5 times the interquartile range from the box. Circles represent individual data points. For 21 patients, histological staging according to METAVIR was available. For 20 patients, data from TE measurements were available ( $F 0-F 2, n=9 ; F 3, F 4, n=12, n=11$ for fibroscan, respectively). MFAP4, microfibrillar-associated protein 4; TE, transient elastography; METAVIR, scoring system for the assessment of hepatic fibrosis; F, fibrosis stage; n.S., not significant. "Significant on Wilcoxon test $(P$-value $<0.05)$. 
tant from the FIB-4 lower cut-off of $1.45 .^{30}$

\section{Serum levels of MFAP4 at baseline, EoT and FU time points}

The changes in MFAP4 serum levels between the baseline, the EoT and the FU decreased in all patients (Fig. 4). This observation was statistically highly significant between $\mathrm{BL}$ and $\mathrm{FU}$ as well as between EoT and FU time points (Table 3). When analyzing patients with F0-F2 and F3, F4 independent of each other it was apparent that the MFAP4 decrease was more pronounced in patients with no to moderate fibrosis (F0-F2). This was also reflected by the statistical analysis because changes in MFAP4 levels in F3 and F4 were not found to be significant.

\section{DISCUSSION}

The disease progression of HCV infection is characterized by the development of hepatic fibrosis, with a highly variable course that ranges from minimal histological impairments to extensive fibrosis with or without the development of hepatocellular carcinoma (HCC). ${ }^{31}$ Although in the last decade the therapy of the HCV infection was revolutionized with the development of DAAs ${ }^{13-16}$ (highly tolerable, cure rates of $>90 \%$ ) up to now one of the most common reasons for liver transplantation still is liver failure as a consequence of HCV infection. ${ }^{32}$ It is well known that the pathways leading to liver injury can be immune system mediated reactions and on the other hand direct viral cytopathic effects. ${ }^{33}$

The liver biopsy is considered the gold standard for assessment

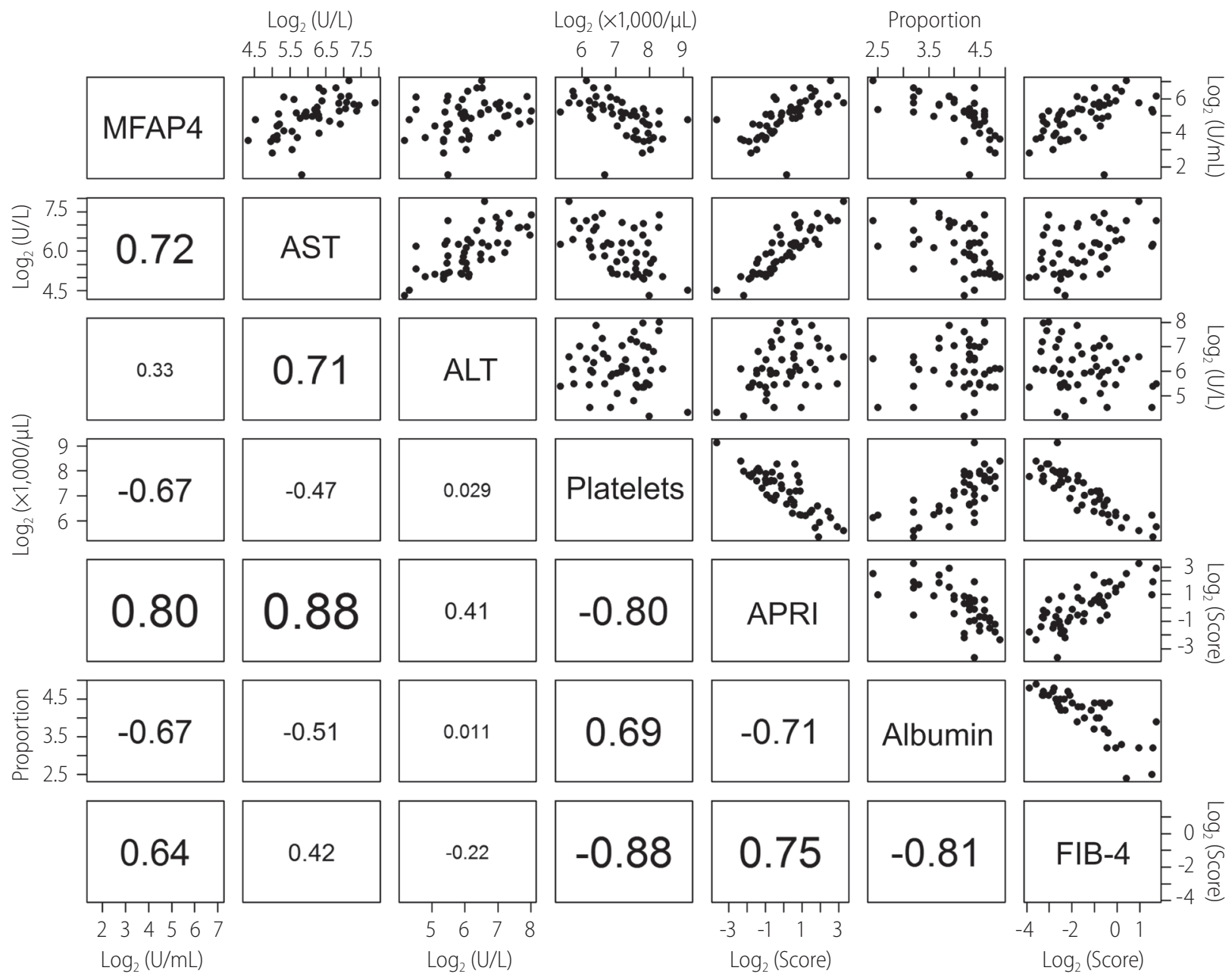

Figure 2. Correlation matrix of baseline MFAP4, APRI, laboratory parameters, and FIB-4. The lower left corner of the figure shows $R^{2}$ values calculated according to the Spearman method (font size corresponds to the quality of the $R^{2}$ value). The upper right half of the figure shows the corresponding scatter plots. Black points represent individual data points. MFAP4, microfibrillar-associated protein 4; AST, aspartate aminotransferase; ALT, alanine aminotransferase; APRI, AST to platelet ratio index; FIB-4, fibrosis-4 score. 

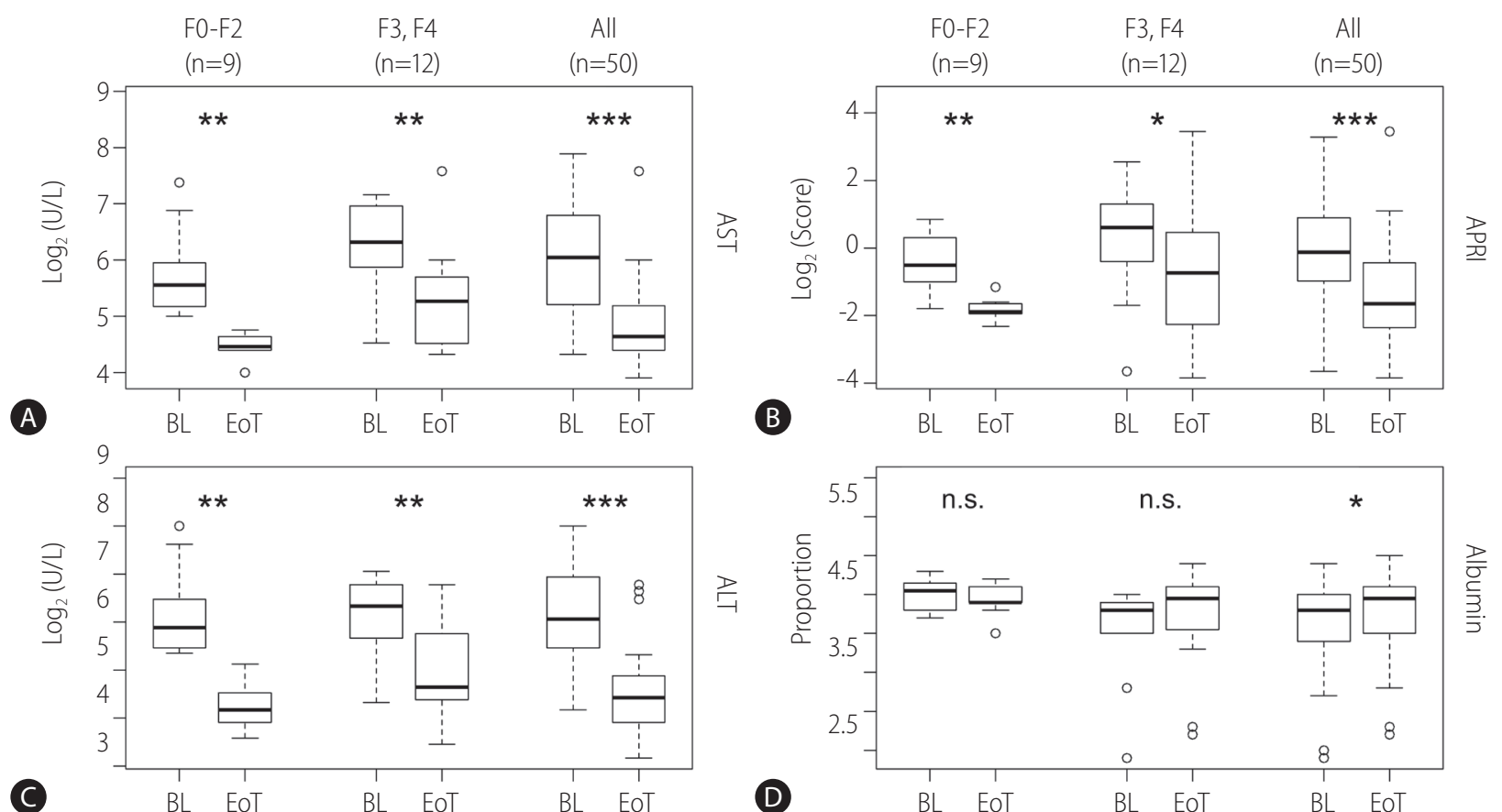

C 10

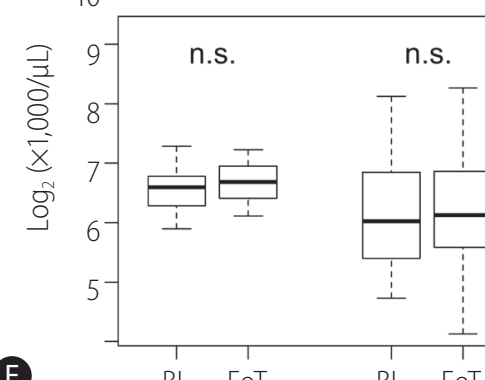

BL EOT

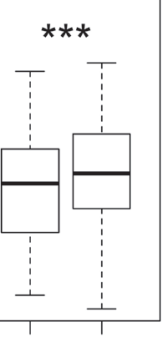

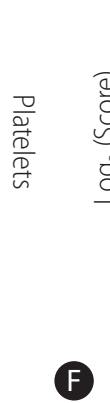

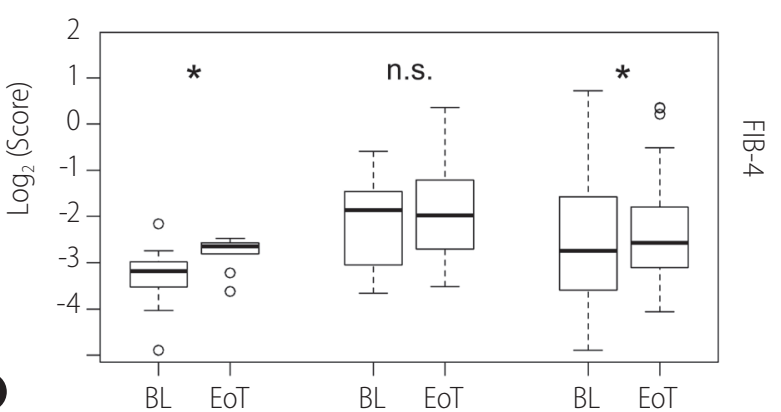

Figure 3. Pairwise comparisons of baseline (BL) and end-of-therapy (EoT) laboratory parameters (A: aspartate aminotransferase [AST]; C: alanine aminotransferase [ALT]; D: Albumin; E: Platelets), AST to platelet ratio index (APRI) (B), and fibrosis-4 score (FIB-4) (F). The upper and lower bounds of boxes represent the first and third quartile per group. Whiskers extend to the most extreme data point, which is no more than 1.5 times the interquartile range from the box. Circles represent individual data points. For 21 patients, histological staging according to METAVIR was available. Those patients were analyzed separately and divided into no to moderate (F0-F2) and severe fibrosis groups. Asterisks indicate significance on Wilcoxon test ( ${ }^{*} P$-value: $<0.05$, "'P-value: $<0.01$, "**" P-value: $<0.001$ ). F, fibrosis stage; n.s., not significant; METAVIR, scoring system for the assessment of hepatic fibrosis.

Table 1. Results of pairwise comparisons of baseline (BL) and end-of-therapy (EoT) laboratory parameters

\begin{tabular}{|c|c|c|c|}
\hline & & Vilcoxon $P$-valu & \\
\hline & F0-F2 $(n=9)^{*}$ & F3, F4 (n=12) & All $(n=50)$ \\
\hline APRI & 0.0039 & 0.0122 & $2.37 \cdot 10^{-8}$ \\
\hline AST & 0.0039 & 0.0093 & $1.45 \cdot 10^{-11}$ \\
\hline ALT & 0.0039 & 0.0068 & $3.89 \cdot 10^{-9}$ \\
\hline Platelets & 0.1641 & 0.5633 & $9.65 \cdot 10^{-6}$ \\
\hline Albumin & 0.8918 & 0.1412 & 0.0026 \\
\hline FIB-4 & 0.0391 & 0.3394 & 0.0257 \\
\hline
\end{tabular}

APRI, aspartate aminotransferase (AST) to platelet ratio index; ALT, alanine aminotransferase; FIB-4, fibrosis-4 score; F, fibrosis stage.

*For $n=21$ patients histological staging of hepatic fibrosis according to scoring system for the assessment of hepatic fibrosis (METAVIR) was available. Patients were divided into no to moderate fibrosis stages (F0-F2) and severe fibrosis and cirrhosis (F3, F4) and analyzed separately. 
Table 2. Median and interquartile range for MFAP4 and laboratory parameters at baseline, the end-of-therapy, and follow-up (only MFAP4)

\begin{tabular}{|c|c|c|c|c|c|c|}
\hline & \multicolumn{2}{|r|}{ Baseline } & \multicolumn{2}{|c|}{ End-of-therapy } & \multicolumn{2}{|r|}{ Follow-up } \\
\hline & Median & $\begin{array}{c}\text { Interquartile range } \\
\text { (Q3-Q1) }\end{array}$ & Median & $\begin{array}{l}\text { Interquartile range } \\
\text { (Q3-Q1) }\end{array}$ & Median & $\begin{array}{c}\text { Interquartile range } \\
\text { (Q3-Q1) }\end{array}$ \\
\hline MFAP4 (U/mL) & 33.05 & 33.7 & 29.6 & 27.2 & 23.2 & 23.2 \\
\hline AST (U/L) & 66 & 74 & 25 & 15.5 & & \\
\hline $\mathrm{ALT}(\mathrm{U} / \mathrm{L})$ & 67 & 79 & 21.5 & 14.5 & & \\
\hline Platelets $(\times 1,000 / \mu \mathrm{L})$ & 151 & 140 & 170 & 157 & & \\
\hline APRI & 0.92 & 1.35 & 0.32 & 0.56 & & \\
\hline Albumin (proportion) & 4.3 & 0.6 & 4.45 & 0.6 & & \\
\hline FIB-4 & 0.30 & 0.49 & 0.34 & 0.34 & & \\
\hline
\end{tabular}

MFAP4, microfibrillar-associated protein 4; AST, aspartate aminotransferase; ALT, alanine aminotransferase; APRI, AST to platelet ratio index; FIB-4, fibrosis-4 score; Q1, first quartile; Q3, third quartile.

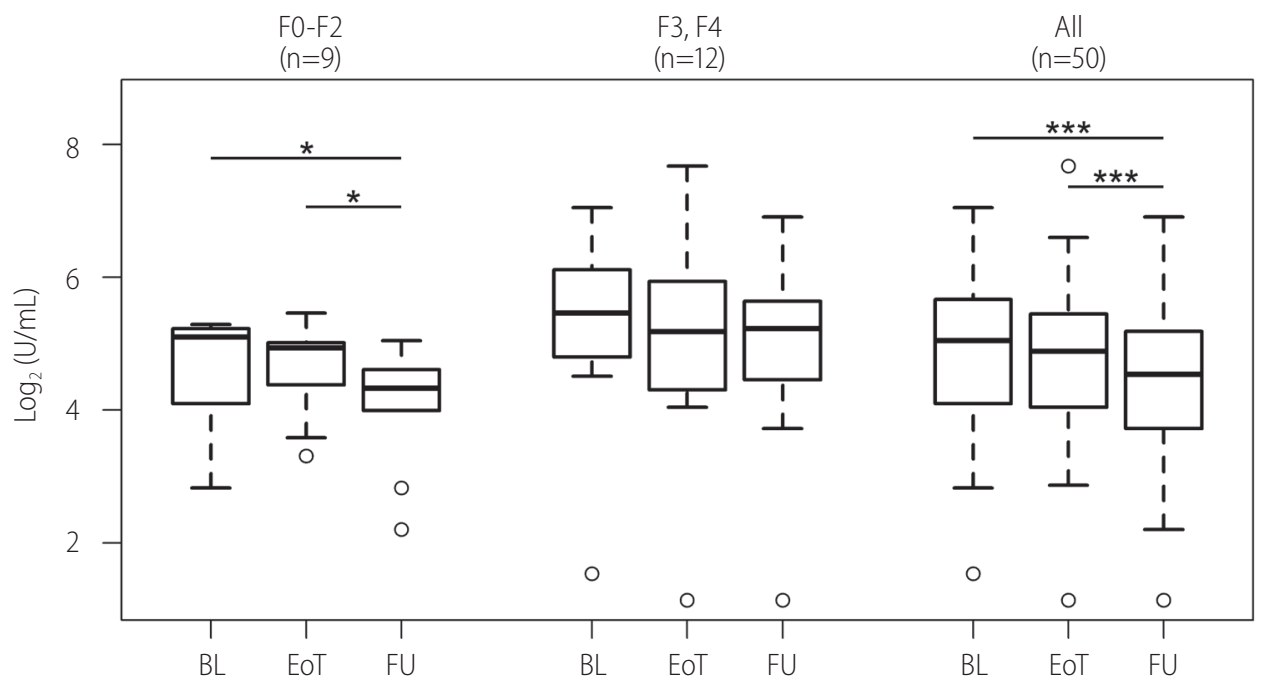

Figure 4. Comparisons of MFAP4 levels at baseline (BL), end-of-therapy (EoT), and follow-up (FU). The upper and lower bounds of boxes represent the first and third quartile per group. Whiskers extend to the most extreme data point, which is no more than 1.5 times the interquartile range from the box. Circles represent individual data points. For 21 patients, histological staging according to METAVIR was available. Those patients were analyzed separately and divided into no to moderate (F0-F2) and severe fibrosis groups. Asterisks indicate significance on pairwise Wilcoxon test after a signifcant Friedman $P$-value ( $P$-value: $<0.05,{ }^{* * *} P$-value: $\left.<0.001\right)$. MFAP4, microfibrillar-associated protein 4; METAVIR, scoring system for the assessment of hepatic fibrosis; F, fibrosis stage.

Table 3. Friedman test with subsequent pairwise Wilcoxon test for baseline, end-of-therapy and follow-up levels of MFAP4

\begin{tabular}{lcccc} 
& Friedman $P$-value & Baseline vs. End-of-therapy & Baseline vs. Follow up & End-of-therapy vs. Follow up \\
\hline F0-F2 $(n=9)$ & 0.01312 & 1 & 0.02344 & 0.02344 \\
F3, F4 $(n=12)$ & $0.09426^{*}$ & - & - & - \\
All $(n=50)$ & 0.00014 & 0.13092 & 0.00007 & 0.00016 \\
\hline
\end{tabular}

MFAP4, microfibrillar-associated protein 4; F, fibrosis stage.

"Pairwise Wilcoxon tests were only performed in case of a significant Friedman $P$-value.

of hepatic fibrosis although the procedure is invasive and carries a significant rate of complications, especially in patients with coagulation disorders. For this reason after pretherapeutic biopsy and subsequently SVR there is usually no monitoring of hepatic fibrosis development in a longitudinal manner. In consequence several non-invasive tests like APRI and FIB-4 have been used to assess 
the residual fibrosis in patients after SVR but the findings were largely different from those received from post-treatment liver biopsy. ${ }^{34}$ This can be explained by the absence of the inflammatory viral stimulus leading to a spontaneous normalization of AST and ALT levels with the eradication of HCV. This rapid decrease of AST, ALT and consequently also APRI has also been observed in our study (Fig. 3). In additon, Sebastiani et al. demonstrated a poor performance of APRI, AST to ALT ratio, Forns' Index, Fibrotest and Fibroindex in 80 viremic patients with persistently normal transaminase values ${ }^{35}$ and therefore concludes that for this group of patients liver biopsy is still necessary. The FIB-4 which also considers AST, ALT and the platelet count was primarily designed to detect high risk patients suffering of advanced fibrosis to avoid unnecessary biopsies. ${ }^{30}$ The predictive power of FIB-4 is limited regarding lower stages of fibrosis and, in our study, correlates primarily with the platelet count at BL. For the comparison between BL and EoT FIB-4 shows unclear results which are contradictory when assessing higher and lower fibrosis stages independently (Fig. 3). Currently published data suggests that the viral eradication leads to a reduction of hepatic fibrosis by demonstrating an improvement of liver stiffness in $\mathrm{TE}$, probably due to reduced necroinflammation and fibrogenesis. ${ }^{17}$ In order to appraise an accurate prognosis and to optimize clinical management these results underline that a valid and preferably non-invasive and everywhere available assessment of residual cirrhosis in HCV patients with an SVR is urgently required. Besides, TE is not nation-wide disposable. Therefore, it is currently difficult to reliably evaluate serial changes in liver fibrosis before and after the eradication of HCV, observing each patient in a longitudinal manner concerning its individual hepatic fibrogenesis. In the present study, we observed that at BL, MFAP4 also correlates with several laboratory parameters, especially AST and APRI. On the other hand, correlation with FIB-4 and also the platelet count, which is considered by both, APRI and FIB-4, was rather weak (Fig. 2). However, in contrast to the prominent drop observed in AST and ALT levels, the decrease in MFAP4 levels was weaker from BL to EOT but sustained until the FU time point (Fig. 4). This illustrates that MFAP4 levels do only weakly represent of the ongoing inflammation in the liver but presumably correlate with tissue remodeling taking place after viral eradication.

The long-standing dogma that cirrhosis is not reversible was disproved by several studies demonstrating that HCV patients with a SVR to IFN-based regimens showed cirrhosis regression in $30 \%$ to $60 \%$ of patients 3 to 5 years after SVR. ${ }^{36-40}$ To date, no data is available for the developing of direct serum markers in chronic HCV patients undergoing therapy with DAAs. ${ }^{41}$ For this reason, we examined the applicability of MFAP4 as a serum biomarker for hepatic fibrosis in chronic HCV patients undergoing therapy with DAAs in this study. It was revealed that serum levels of MFAP4 decrease during the DAA-based HCV therapy and more clearly after therapy until the follow-up time point (Fig. 4). A dramatic decrease in inflammatory activity was represented by APRI and the corresponding laboratory parameters AST and ALT (Fig. 3). In addition an improvement in liver synthesis was shown by an increase in serum albumin levels in patients with SVR. These findings are direct results of DAA-based viral eradication and not influenced by the well-known antifibrotic effects of IFN based HCV therapies. Whether this observation reflects a reduced fibrogensis as a result of viral eradication and therefore a diminished fibrogenic stimulus in the liver or may even indicate a regression of hepatic fibrosis cannot be finally clarified in the absence of corresponding liver biopsies. Other helpful parameters like TE or fibrotest were unfortunately not available for EoT and FU or the whole cohort at BL. This represents a shortcoming of our study and has to be considered when evaluating our data and needs to be addressed in further studies. It is evident that regression of hepatic fibrosis is associated with a reduced risk of developing HCC, hepatic decompensation and variceal bleeding, while, on the contrary, a clinical benefit was not observed in SVR patients with residual cirrhosis. ${ }^{42}$ In consequence, all SVR patients with advanced hepatic fibrosis are currently recommended to keep lifelong surveillance for HCC with abdominal ultrasound every 6 months ${ }^{43}$ as these patients still carry a $0.6-1.2 \%$ yearly risk of HCC. ${ }^{44,45}$ In the present study, we could show that MFAP4 as a non-invasive biomarker might have the potential to be a valuable tool (e.g. in a future multi-biomarker setting) for the assessment of hepatic fibrosis after eradication of HCV. Thus, MFAP4 could be helpful for the identification and monitoring of HCV patients with advanced hepatic fibrosis. It must be emphasized once more that complications (e.g. HCC, hepatic decompensation etc.) of the HCV infection occur even after SVR and clarify the need of an effective risk management in this special cohort of patients.

\section{Authors' contribution}

Christian Mölleken designed research, analyzed data and wrote the manuscript.

Maike Ahrens analyzed data and wrote the manuscript.

Anders Schlosser analyzed samples.

Julia Dietz collected and provided samples and data.

Martin Eisenacher analyzed data. 
Helmut E. Meyer designed research.

Wolff Schmiegel designed research.

Uffe Holmskov analyzed samples and designed research.

Christoph Sarrazin collected and provided samples and data.

Grith Lykke Sorensen analyzed samples and designed research.

Barbara Sitek designed research, analyzed data and wrote the manuscript.

Thilo Bracht designed research, analyzed data and wrote the manuscript.

\section{Acknowledgements}

The authors thank Kristin Rosowski, Birgit Korte and Stephanie Tautges for their excellent technical assistance as well as Lukas Funke and Karin Schork for their help with the R scripting.

\section{Finacial support}

A part of this study was funded by P.U.R.E. (Protein Unit for Research in Europe), a project of North Rhine-Westphalia, a federal state of Germany and by de.NBI, a project of the Federal Ministry of Education and Research (BMBF) (FKZ 031 A 534 A).

\section{Conflicts of Interest}

The authors have no conflicts of interest to disclose.

\section{SUPPLEMENTARY MATERIALS}

Supplementary materials are available at Clinical and Molecular Hepatology website (http://www.e-cmh.org).

\section{REFERENCES}

1. Gower E, Estes C, Blach S, Razavi-Shearer K, Razavi H. Global epidemiology and genotype distribution of the hepatitis $C$ virus infection. J Hepatol 2014;61(1 Suppl):S45-S57.

2. Lerat $H$, Honda $M$, Beard MR, Loesch $K$, Sun J, Yang $Y$, et al. Steatosis and liver cancer in transgenic mice expressing the structural and nonstructural proteins of hepatitis C virus. Gastroenterology 2002;122:352-365.

3. Perlemuter G, Sabile A, Letteron P, Vona G, Topilco A, Chrétien Y, et al. Hepatitis $C$ virus core protein inhibits microsomal triglyceride transfer protein activity and very low density lipoprotein secretion: a model of viral-related steatosis. FASEB J 2002;16:185-194.

4. Moriya K, Yotsuyanagi H, Shintani Y, Fujie H, Ishibashi K, Matsuura
Y, et al. Hepatitis C virus core protein induces hepatic steatosis in transgenic mice. J Gen Virol 1997;78 (Pt 7):1527-1531.

5. Gong $G$, Waris $G$, Tanveer $R$, Siddiqui A. Human hepatitis $C$ virus NS5A protein alters intracellular calcium levels, induces oxidative stress, and activates STAT-3 and NF-kappa B. Proc Natl Acad Sci U S A 2001;98:9599-9604.

6. Friedman SL. Molecular regulation of hepatic fibrosis, an integrated cellular response to tissue injury. J Biol Chem 2000;275:2247-2250.

7. Bissell DM. Chronic liver injury, TGF-beta, and cancer. Exp Mol Med 2001:33:179-190.

8. Gressner AM, Weiskirchen R, Breitkopf K, Dooley S. Roles of TGFbeta in hepatic fibrosis. Front Biosci 2002;7:d793-d807.

9. McCrudden R, Iredale JP. Liver fibrosis, the hepatic stellate cell and tissue inhibitors of metalloproteinases. Histol Histopathol 2000;15:1159-1168.

10. Knittel T, Kobold D, Saile B, Grundmann A, Neubauer K, Piscaglia $F$, et al. Rat liver myofibroblasts and hepatic stellate cells: different cell populations of the fibroblast lineage with fibrogenic potential. Gastroenterology 1999;117:1205-1221.

11. Suou T, Hosho K, Kishimoto $Y$, Horie $Y$, Kawasaki H. Long-term decrease in serum $\mathrm{N}$-terminal propeptide of type III procollagen in patients with chronic hepatitis $\mathrm{C}$ treated with interferon alfa. Hepatology 1995;22:426-431.

12. Hiramatsu N, Hayashi N, Kasahara A, Hagiwara H, Takehara T, Haruna $Y$, et al. Improvement of liver fibrosis in chronic hepatitis $C$ patients treated with natural interferon alpha. J Hepatol 1995;22:135142.

13. Dore GJ, Ward J, Thursz M. Hepatitis C disease burden and strategies to manage the burden (Guest Editors Mark Thursz, Gregory Dore and John Ward). J Viral Hepat 2014;21 Suppl 1:1-4.

14. Dore GJ, Feld JJ. Hepatitis C virus therapeutic development: in pursuit of "perfectovir". Clin Infect Dis 2015;60:1829-1836.

15. Thomas DL. Curing hepatitis $C$ with pills: a step toward global control. Lancet 2010;376:1441-1442.

16. Hill A, Cooke G. Medicine. Hepatitis C can be cured globally, but at what cost? Science 2014;345:141-142.

17. Knop V, Hoppe D, Welzel T, Vermehren J, Herrmann E, Vermehren A, et al. Regression of fibrosis and portal hypertension in HCV-associated cirrhosis and sustained virologic response after interferon-free antiviral therapy. J Viral Hepat 2016;23:994-1002.

18. Mölleken C, Sitek B, Henkel C, Poschmann G, Sipos B, Wiese S, et al. Detection of novel biomarkers of liver cirrhosis by proteomic analysis. Hepatology 2009;49:1257-1266.

19. Bracht $T$, Schweinsberg V, Trippler M, Kohl M, Ahrens M, Padden $J$, et al. Analysis of disease-associated protein expression using quantitative proteomics--fibulin-5 is expressed in association with hepatic fibrosis. J Proteome Res 2015;14:2278-2286.

20. Wulf-Johansson H, Lock Johansson S, Schlosser A, Trommelholt 
Holm A, Rasmussen LM, Mickley H, et al. Localization of microfibrillar-associated protein 4 (MFAP4) in human tissues: clinical evaluation of serum MFAP4 and its association with various cardiovascular conditions. PloS One 2013;8:e82243.

21. Pilecki B, Holm AT, Schlosser A, Moeller JB, Wohl AP, Zuk AV, et al. Characterization of microfibrillar-associated protein 4 (MFAP4) as a tropoelastin- and fibrillin-binding protein involved in elastic fiber formation. J Biol Chem 2016;291:1103-1114.

22. Schlosser A, Pilecki B, Hemstra LE, Kejling K, Kristmannsdottir GB, Wulf-Johansson $\mathrm{H}$, et al. MFAP4 promotes vascular smooth muscle migration, proliferation and accelerates neointima formation. Arterioscler Thromb Vasc Biol 2016;36:122-133.

23. Pilecki B, Schlosser A, Wulf-Johansson H, Trian T, Moeller JB, Marcussen $\mathrm{N}$, et al. Microfibrillar-associated protein 4 modulates airway smooth muscle cell phenotype in experimental asthma. Thorax 2015;70:862-872.

24. Lock-Johansson S, Vestbo J, Sorensen GL. Surfactant protein D, club cell protein 16, pulmonary and activation-regulated chemokine, Creactive protein, and fibrinogen biomarker variation in chronic obstructive lung disease. Respir Res 2014;15:147.

25. Hemstra LE, Schlosser A, Lindholt JS, Sorensen GL. Microfibrillarassociated protein 4 variation in symptomatic peripheral artery disease. J Transl Med 2018;16:159.

26. Blindbæk SL, Schlosser A, Green A, Holmskov U, Sorensen GL, Grauslund J. Association between microfibrillar-associated protein 4 (MFAP4) and micro- and macrovascular complications in long-term type 1 diabetes mellitus. Acta Diabetol 2017;54:367-372.

27. Sækmose SG, Mössner B, Christensen PB, Lindvig K, Schlosser A, Holst $R$, et al. Microfibrillar-associated protein 4: a potential biomarker for screening for liver fibrosis in a mixed patient cohort. Plos One 2015;10:e0140418.

28. Bracht T, Mölleken C, Ahrens M, Poschmann G, Schlosser A, Eisenacher $M$, et al. Evaluation of the biomarker candidate MFAP4 for non-invasive assessment of hepatic fibrosis in hepatitis C patients. J Transl Med 2016;14:201.

29. Sækmose SG, Schlosser A, Holst R, Johansson SL, Wulf-Johansson $H$, Tornøe I, et al. Enzyme-linked immunosorbent assay characterization of basal variation and heritability of systemic microfibrillarassociated protein 4. PloS One 2013;8:e82383.

30. Sterling RK, Lissen E, Clumeck N, Sola R, Correa MC, Montaner J, et al. Development of a simple noninvasive index to predict significant fibrosis in patients with HIV/HCV coinfection. Hepatology 2006:43:1317-1325.

31. Alberti A, Chemello L, Benvegnù L. Natural history of hepatitis C. J Hepatol 1999;31 Suppl 1:17-24.

32. Ghany MG, Strader DB, Thomas DL, Seeff LB; American Association for the Study of Liver Diseases. Diagnosis, management, and treatment of hepatitis C: an update. Hepatology 2009;49:1335-1374.
33. Mengshol JA, Golden-Mason L, Rosen HR. Mechanisms of disease: HCV-induced liver injury. Nat Clin Pract Gastroenterol Hepatol 2007:4:622-634

34. D'Ambrosio R, Degasperi E, Aghemo A, Fraquelli M, Lampertico P, Rumi MG, et al. Serological tests do not predict residual fibrosis in hepatitis $C$ cirrhotics with a sustained virological response to interferon. PloS One 2016;11:e0155967.

35. Sebastiani G, Vario A, Guido M, Alberti A. Performance of noninvasive markers for liver fibrosis is reduced in chronic hepatitis $C$ with normal transaminases. J Viral Hepat 2008;15:212-218.

36. Reichard O, Glaumann H, Frydén A, Norkrans G, Wejstål R, Weiland $\mathrm{O}$. Long-term follow-up of chronic hepatitis $C$ patients with sustained virological response to alpha-interferon. J Hepatol 1999:30:783-787.

37. Shiratori Y, Imazeki F, Moriyama M, Yano M, Arakawa Y, Yokosuka O, et al. Histologic improvement of fibrosis in patients with hepatitis $C$ who have sustained response to interferon therapy. Ann Intern Med 2000;132:517-524.

38. Poynard T, McHutchison J, Manns M, Trepo C, Lindsay K, Goodman $\mathrm{Z}$, et al. Impact of pegylated interferon alfa- $2 \mathrm{~b}$ and ribavirin on liver fibrosis in patients with chronic hepatitis C. Gastroenterology 2002;122:1303-1313.

39. Arif A, Levine RA, Sanderson SO, Bank L, Velu RP, Shah A, et al. Regression of fibrosis in chronic hepatitis $C$ after therapy with interferon and ribavirin. Dig Dis Sci 2003;48:1425-1430.

40. Pol S, Carnot F, Nalpas B, Lagneau JL, Fontaine H, Serpaggi J, et al. Reversibility of hepatitis $C$ virus-related cirrhosis. Hum Pathol 2004;35:107-112.

41. Valva P, Ríos DA, De Matteo E, Preciado MV. Chronic hepatitis C virus infection: serum biomarkers in predicting liver damage. World J Gastroenterol 2016;22:1367-1381.

42. Mallet V, Gilgenkrantz $H$, Serpaggi J, Verkarre V, Vallet-Pichard A, Fontaine $\mathrm{H}$, et al. Brief communication: the relationship of regression of cirrhosis to outcome in chronic hepatitis C. Ann Intern Med 2008;149:399-403.

43. European Association for the Study of the Liver; European Organisation for Research and Treatment of Cancer. EASL-EORTC clinical practice guidelines: management of hepatocellular carcinoma. J Hepatol 2012;56:908-943.

44. Aleman S, Rahbin N, Weiland O, Davidsdottir L, Hedenstierna M, Rose $N$, et al. A risk for hepatocellular carcinoma persists longterm after sustained virologic response in patients with hepatitis Cassociated liver cirrhosis. Clin Infect Dis 2013;57:230-236.

45. Cardoso AC, Moucari R, Figueiredo-Mendes C, Ripault MP, Giuily $N$, Castelnau $C$, et al. Impact of peginterferon and ribavirin therapy on hepatocellular carcinoma: incidence and survival in hepatitis $C$ patients with advanced fibrosis. J Hepatol 2010;52:652-657. 